\title{
Facetas diretas anteriores: Uma revisão de literatura
}

\author{
Direct veneers anteriors: A literature review \\ Facetas directas anteriores: Una revisión de la literatura
}

\author{
Karllos Matheus Gonçalves de Campos \\ ORCID: https://orcid.org/0000-0001-7005-0642 \\ Universidade Federal de Campina Grande, Brasil \\ E-mail: karlos_campos@hotmail.com \\ Rodrigo Araújo Rodrigues \\ ORCID: https://orcid.org/0000-0001-8457-4084 \\ Universidade Federal de Campina Grande, Brasil \\ E-mail: rodrigo.protesedental@gmail.com \\ Camila Helena Machado da Costa Figueiredo \\ ORCID: https://orcid.org/0000-0002-1340-4042 \\ Universidade Federal de Campina Grande, Brasil \\ E-mail: camila_helena_@hotmail.com \\ Gymenna Maria Tenório Guenes \\ ORCID: https://orcid.org/0000-0002-5447-0193 \\ Universidade Federal de Campina Grande, Brasil \\ E-mail: gymennat@yahoo.com.br \\ Maria Angélica Sátyro Gomes Alves \\ ORCID: https://orcid.org/0000-0003-3329-8360 \\ Universidade Federal de Campina Grande, Brasil \\ E-mail: angelicasatyro@hotmail.com \\ Rosana Araújo Rosendo \\ ORCID: https://orcid.org/0000-0002-3795-8832 \\ Universidade Federal de Campina Grande, Brasil \\ E-mail: cesprodonto@hotmail.com \\ Elizandra Silva da Penha \\ ORCID: https://orcid.org/0000-0001-6264-5232 \\ Universidade Federal de Campina Grande, Brasil \\ E-mail: elizandrapenha@ hotmail.com \\ Paula Lima Nogueira \\ ORCID: https://orcid.org/0000-0001-7425-3201 \\ Universidade Federal de Campina Grande, Brasil \\ E-mail: paulalimanogueira@hotmail.com \\ Maria Vitoria Oliveira Dantas \\ ORCID: https://orcid.org/0000-0001-7663-3793 \\ Universidade Federal de Campina Grande, Brasil \\ E-mail:mvitoria.od@hotmail.com \\ Luanna Abílio Diniz Melquiades de Medeiros \\ ORCID: https://orcid.org/ 0000-0002-1630-3968 \\ Universidade Federal de Campina Grande, Brasil \\ E-mail: luannaabiliod@gmail.com
}

\section{Resumo}

A procura por uma estética dental agradável e harmoniosa tem proporcionado um aumento significante nas pesquisas de novos materiais e técnicas de restauração direta que otimizem a qualidade, atingindo padrões quase que imperceptíveis da interface dente-restauração, além de devolver a forma e função do elemento, com menor desgaste da estrutura dental, quando comparado aos meios indiretos. Nesse sentido, o objetivo desse estudo foi realizar uma revisão bibliográfica da literatura sobre facetas diretas anteriores expondo suas vantagens e limitações em comparação com as facetas indiretas. Foram analisados artigos publicados no LILLACS (Literatura Latino-Americana e do Caribe em Ciências da Saúde), BBO-ODONTOLOGIA (Bibliografia Brasileira de Odontologia), MEDLINE (Medical Literature Analysis and Retrieval System Online) e PUBMED (National Library of Medicine). Por intermédio dos resultados obtidos nesta revisão da literatura, onde 14 (quatorze) artigos ficaram para compor o estudo, foi possível verificar que através do aperfeiçoamento dos sistemas adesivos e das resinas compostas as facetas diretas podem restabelecer a cor, a forma e a funcionalidade dental, permitindo reabilitar estético-funcionalmente os elementos dentários, harmonizando-os com os demais componentes orofaciais do paciente, mas é imprescindível que se faça um correto diagnóstico e aplique uma boa técnica para que consiga ter um resultado satisfatório. Conclui-se que facetas diretas em resina composta são eficientes para reabilitar elementos dentários, permitindo estética agradável, bem 
como a função adequada. Sendo consideradas um procedimento conservador, de menor custo e mais rápido quando comparadas às facetas indiretas em cerâmica.

Palavras-chave: Facetas diretas; Dentes anteriores; Estética dental; Resina composta.

\begin{abstract}
The search for a pleasant and harmonious dental aesthetic has led to a significant increase in research on new materials and techniques for direct restorations that optimize the quality, achieving almost imperceptible patterns of the tooth-restoration interface, as well as restoring the shape and function of the tooth with less wear of the dental structure when compared to indirect means. In this sense, the aim of this study was to perform a literature review on anterior direct veneers exposing their advantages and limitations compared to indirect veneers. Articles published in LILLACS (Latin American and Caribbean Literature on Health Sciences), BBO-ODONTOLOGIA (Brazilian Bibliography of Dentistry), MEDLINE (Medical Literature Analysis and Retrieval System Online) and PUBMED (National Library of Medicine) were analyzed. Through the results obtained in this literature review, where 14 (fourteen) articles were selected for the study, it was possible to verify that through the improvement of adhesive systems and composite resins, direct veneers can restore tooth color, shape and functionality, allowing aesthetic and functional rehabilitation of the dental elements, harmonizing them with the other orofacial components of the patient, but it is essential to make a correct diagnosis and apply a good technique to achieve a satisfactory result. We conclude that direct composite resin veneers are efficient for rehabilitating dental elements, allowing a pleasant esthetic, as well as adequate function. They are considered a conservative procedure, lower cost and faster when compared to indirect ceramic veneers.
\end{abstract}

Keywords: Direct facets; Anterior teeth; Dental aesthetics; Composite resin.

\title{
Resumen
}

La búsqueda de una estética dental agradable y armoniosa ha proporcionado un aumento significativo en la búsqueda de nuevos materiales y técnicas de restauración directa que optimicen la calidad, alcanzando estándares casi imperceptibles de la interfaz diente-restauración, además de devolver la forma y función del elemento. con menor desgaste de la estructura dental, en comparación con medios indirectos. En este sentido, el objetivo de este estudio fue realizar una revisión bibliográfica de la literatura sobre facetas directas anteriores, exponiendo sus ventajas y limitaciones en comparación con las facetas indirectas. Se analizaron los artículos publicados en LILLACS (Literatura Latinoamericana y del Caribe en Ciencias de la Salud), BBO-ODONTOLOGIA (Bibliografía de Odontología Brasileña), MEDLINE (Sistema de Análisis y Recuperación de Literatura Médica en Línea) y PUBMED (Biblioteca Nacional de Medicina). Mediante los resultados obtenidos en esta revisión de la literatura, donde quedaron 14 (catorce) artículos para componer el estudio, se pudo verificar que a través de la mejora de los sistemas adhesivos y resinas compuestas, las facetas directas pueden restaurar el color, la forma y la funcionalidad dental, permitiendo la Rehabilitación estética-funcional de los elementos dentarios, armonizándolos con los demás componentes orofaciales del paciente, pero es fundamental hacer un correcto diagnóstico y aplicar una buena técnica para que se pueda tener un resultado satisfactorio. Se concluye que las facetas directas en resina compuesta son eficientes para rehabilitar elementos dentarios, permitiendo una estética agradable, así como el correcto funcionamiento. Siendo considerado un procedimiento conservador, de menor costo y más rápido en comparación con las facetas cerámicas indirectas.

Palabras clave: Facetas directas; Dientes anteriores; Estética dental; Resina compuesta.

\section{Introdução}

Use o parágrafo como modelo A aparência é fator decisivo na autoestima das pessoas, sendo ela importante na busca de procedimentos na odontologia estética, esta última é capaz de tratar ou minimizar assimetrias faciais, problemas periodontais e dentários. Sendo capaz de alterar tamanho, forma, cor e textura dos dentes, deixando-os com aspecto natural (Dos Reis Goyatá et al., 2017).

A procura por uma estética dental agradável e harmoniosa tem proporcionado um aumento significante nas pesquisas de novos materiais e técnicas de restauração direta que otimizem a qualidade, atingindo padrões quase que imperceptíveis da interface dente-restauração, além de devolver a forma e função do elemento, com menor desgaste da estrutura dental, quando comparado aos meios indiretos (Santos et al., 2018).

Considerando que a restauração indireta provoca, na maioria das vezes, um maior desgaste da estrutura dental sadia, comparada à técnica direta, e que a introdução de novos compósitos, cada vez melhores, tem proporcionado previsibilidade, longevidade e qualidade das restaurações, pode-se optar, em alguns casos, por restaurações diretas em resina composta (Pottmaier et al., 2017). 
Sabendo-se que atualmente não existem mais vantagens e limitações totalitárias, a escolha do tratamento depende do conhecimento e domínio das técnicas por parte do cirurgião-dentista e, da qualidade dos materiais utilizados que vão influenciar diretamente no sucesso clínico da restauração (Guerra et al., 2017).

Diante disso, discutir sobre facetas diretas anteriores, justifica-se pelo menor custo destas e maior preservação de estrutura dentária sadia, quando comparadas as restaurações indiretas, a exemplo das facetas indiretas e os laminados cerâmicos.

Mesmo levando em consideração as limitações como o pouco tempo de acompanhamento dos casos clínicos de facetas, possibilitando apenas análise descritiva, notou que é possível observar que resinas compostas possuem desempenho no mínimo semelhante as cerâmicas dentárias com até quatro anos de acompanhamento, sendo que as facetas de resina não apresentaram substituição e menor taxa de reparo em relação às cerâmicas (Patias, 2015).

Diante do exposto, é certo que durante uma avaliação prospectiva de seis meses todas as restaurações foram consideradas clinicamente aceitáveis assim como todos os pacientes mostraram-se inteiramente satisfeitos. Chegando à conclusão que a restauração direta com resina composta é uma técnica segura, e que demonstra uma performance aceitável de avaliação clínica, uma das limitações é que essa técnica necessita de treinamento para desenvolvimento das habilidades e de um olhar mais crítico do operador (Biezus, 2016).

Para tanto, o presente estudo teve como objetivo avaliar as facetas diretas anteriores, bem como identificar as vantagens e limitações destas em relação as facetas indiretas, por intermédio de uma revisão da literatura.

\section{Metodologia}

O presente estudo consiste em uma revisão da literatura de natureza qualitativa, que segundo Polit \& Beck (2011), corresponde a apresentação de novas informações ao disponibilizar conhecimentos atuais sobre o tema abordado ou enfatizar lacunas no corpo de pesquisas, e assim impulsionar pesquisadores a melhorar a base de dados científicos. Neste estudo foram pesquisados artigos científicos a fim de se avaliar as facetas diretas anteriores, bem como identificar as vantagens e limitações destas quando comparadas as facetas indiretas na Odontologia.

Nesse sentido, os resultados foram apresentados de forma qualitativa, onde a pesquisa dos artigos foi realizada nas bases de dados Medline, Lilacs, BBO e PubMed. Com isso, foram usadas as seguintes palavras-chave: "facetas diretas", "dentes anteriores", "estética dental" e "resina composta". Com o objetivo de ampliar a busca, foi utilizado o operador booleano "or".

Com a finalidade de definir claramente a adequação da literatura encontrada para este estudo de revisão, foram estabelecidos os seguintes critérios de inclusão dos estudos: a) estudos com intervenções que apresentassem conteúdos relativos as facetas direta em dentes anteriores, incluindo suas vantagens e limitações; b) ter intervalo de ano de publicação entre 2015 a 2021; c) ter sido realizado no Brasil.

Além de eliminar artigos que não fossem condizentes com os parâmetros listados acima, os critérios de exclusão foram: a) estudos não disponibilizados no formato fulltext; b) estudos duplicados disponíveis em mais de uma base de dado; c) estudos que não condiziam com as palavras-chave; d) trabalhos monográficos, e e) estudos que não abordam a temática.

Na primeira verificação praticada, tendo por base os títulos e os resumos dos artigos, foram rejeitados aqueles que não preenchiam aos critérios de inclusão ou apresentassem algum dos critérios de exclusão. Quando o artigo não pôde ser incluído ou rejeitado com certeza a partir da primeira análise, o texto completo foi selecionado e analisado em um momento posterior.

Os artigos foram lidos e sintetizados, avaliando-se os aspectos de autoria, ano de publicação, objetivo do estudo e metodologia. Após a síntese dos textos, os artigos foram organizados de modo a facilitar a compreensão dos dados e o processo de análise. 


\section{Resultados e Discussão}

A estratégia de pesquisa descrita que utilizou as palavras-chave: “facetas diretas", “dentes anteriores", "estética dental" e "resina composta". No período específico de 2015 a 2021, publicados em Português, encontrou 48 artigos científicos na base de dados Lilacs, Medline, BBO e PubMed. Analisando a partir dos critérios de inclusão e exclusão, foram descartados 05 por não estarem disponibilizados no formato fulltext, 09 estudos que não condiziam com as palavras-chave, 08 por não abordarem a temática, 10 artigos duplicados e 02 por serem teses monográficas. Com isso, após a leitura criteriosa dos artigos, apenas 14 artigos foram selecionados por cumprirem os critérios propostos, podendo ser visto na Figura 01.

Figura 1: Esquema das etapas de seleção dos artigos.

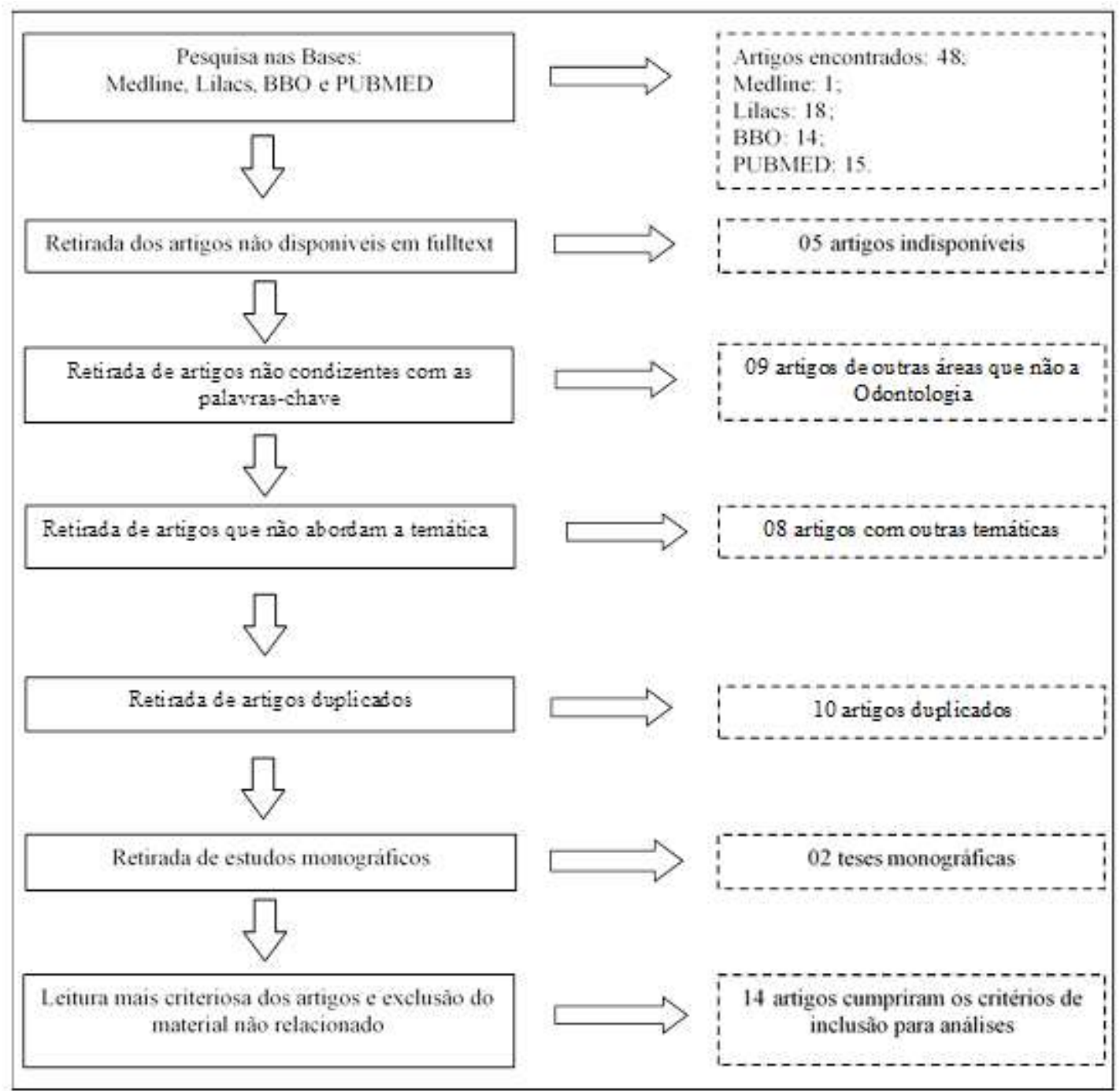

Fonte: Autores (2021).

No Quadro 1 encontram-se, respectivamente, a categorização dos artigos recuperados quanto aos autores, título do artigo, objetivo do artigo, tipo de pesquisa, e ano de publicação. 
Quadro 1: Artigos relacionados as "Facetas diretas anteriores".

\begin{tabular}{|c|c|c|c|c|}
\hline Autores & Título do Artigo & Objetivo do Artigo & $\begin{array}{l}\text { Tipo de } \\
\text { Pesquisa }\end{array}$ & Ano \\
\hline $\begin{array}{c}\text { MACHADO, } \\
\text { Alexandre Costa et al. }\end{array}$ & $\begin{array}{l}\text { Reabilitação estética e funcional } \\
\text { com facetas diretas após histórico } \\
\text { de traumatismo dento-alveolar }\end{array}$ & $\begin{array}{l}\text { Detalhar as etapas clínicas de reabilitação estética } \\
\text { e funcional com facetas diretas em resina } \\
\text { composta em incisivos centrais com histórico de } \\
\text { traumatismo dento-alveolar. }\end{array}$ & $\begin{array}{l}\text { Relato de } \\
\text { Caso }\end{array}$ & 2016 \\
\hline $\begin{array}{l}\text { MIRANDA, Rafael } \\
\text { Resende } \text { et al. }\end{array}$ & $\begin{array}{c}\text { Tratamento odontológico } \\
\text { integrado com ênfase em estética }\end{array}$ & $\begin{array}{l}\text { Descrever as etapas de diagnóstico, } \\
\text { planejamento, tratamento e transformação de um } \\
\text { sorriso. }\end{array}$ & $\begin{array}{l}\text { Relato de } \\
\text { Caso }\end{array}$ & 2016 \\
\hline $\begin{array}{c}\text { ALVARENGA, } \\
\text { Daniela Batista et al. }\end{array}$ & $\begin{array}{c}\text { Inter-relação } \\
\text { periodontia/dentística na correção } \\
\text { de sorriso gengival }\end{array}$ & $\begin{array}{l}\text { Relatar um caso clínico de planejamento e } \\
\text { correção de sorriso gengival com cirurgia plástica } \\
\text { periodontal e facetas de resina composta. }\end{array}$ & $\begin{array}{l}\text { Relato de } \\
\text { Caso }\end{array}$ & 2018 \\
\hline $\begin{array}{c}\text { REIS, Giselle } \\
\text { Rodrigues et al. }\end{array}$ & $\begin{array}{l}\text { Mock-up: previsibilidade e } \\
\text { facilitador das restaurações } \\
\text { estéticas em resina composta }\end{array}$ & $\begin{array}{l}\text { Apresentar relato de caso clínico mostrando a } \\
\text { importância do planejamento reverso na } \\
\text { reabilitação estética, realizada por meio de } \\
\text { facetas diretas em resina composta. }\end{array}$ & $\begin{array}{l}\text { Relato de } \\
\text { Caso }\end{array}$ & 2018 \\
\hline $\begin{array}{l}\text { ARAÚJO, Isabela } \\
\text { Dantas Torres et al. }\end{array}$ & $\begin{array}{l}\text { Reabilitação estética anterior com } \\
\text { resina composta }\end{array}$ & $\begin{array}{c}\text { Apresentar um caso clínico cujo plano de } \\
\text { tratamento foi composto pela confecção de } \\
\text { facetas diretas em resina composta dos elementos } \\
\text { antero-superiores a fim de devolver a estética } \\
\text { adequada para a paciente. }\end{array}$ & $\begin{array}{l}\text { Relato de } \\
\text { Caso }\end{array}$ & 2019 \\
\hline $\begin{array}{l}\text { BAPTISTA, Isabela } \\
\text { Ardenghi et al. }\end{array}$ & $\begin{array}{l}\text { Associação de diferentes } \\
\text { cirurgias periodontais como } \\
\text { alternativa de restabelecimento } \\
\text { de harmonia gengival }\end{array}$ & $\begin{array}{l}\text { Apresentar um relato de caso no qual a paciente } \\
\text { se queixava de desarmonia do sorriso, desta } \\
\text { forma o aumento de coroa clínica estética e o } \\
\text { retalho reposicionado coronalmente, foram } \\
\text { escolhidos juntamente com a reanatomização em } \\
\text { resina composta, para restabelecer a harmonia } \\
\text { gengival e dentária. }\end{array}$ & $\begin{array}{l}\text { Relato de } \\
\text { Caso }\end{array}$ & 2019 \\
\hline $\begin{array}{l}\text { ARAÚJO, Isabela } \\
\text { Coelho et al. }\end{array}$ & $\begin{array}{l}\text { Restaurações estéticas em } \\
\text { substratos com alteração } \\
\text { cromática }\end{array}$ & $\begin{array}{l}\text { Facetamento vestibular direto e indireto de dentes } \\
\text { anteriores, empregando resina composta e } \\
\text { cerâmica odontológica, realizando uma análise } \\
\text { comparativa entre ambas as técnicas } \\
\text { restauradoras para que os profissionais possam } \\
\text { compreender a indicação, vantagem, } \\
\text { desvantagem e contraindicação de tais } \\
\text { modalidades restauradoras, visando alcançar o } \\
\text { sucesso do tratamento e a satisfação do paciente. }\end{array}$ & $\begin{array}{l}\text { Relato de } \\
\text { Caso }\end{array}$ & 2019 \\
\hline
\end{tabular}


Research, Society and Development, v. 10, n. 6, e48910615729, 2021

(CC BY 4.0) | ISSN 2525-3409 | DOI: http://dx.doi.org/10.33448/rsd-v10i6.15729

\begin{tabular}{|c|c|c|c|c|}
\hline $\begin{array}{l}\text { CARRIJO, Denise } \\
\text { Jesus et al. }\end{array}$ & $\begin{array}{c}\text { Restaurações Estéticas Anteriores } \\
\text { Diretas e Indiretas: } \\
\text { Revisão de Literatura }\end{array}$ & $\begin{array}{l}\text { Abordar sobre restaurações diretas em resina } \\
\text { composta e indiretas em cerâmica realizadas em } \\
\text { dentes anteriores apontando suas principais } \\
\text { vantagens, desvantagens fatores de sucesso e } \\
\text { principais motivos de falha que irão influir sobre } \\
\text { sua longevidade clínica. }\end{array}$ & $\begin{array}{l}\text { Revisão } \\
\text { Bibliográfic } \\
\text { a }\end{array}$ & 2019 \\
\hline $\begin{array}{c}\text { DEMARCO, Flávio } \\
\text { Fernando et al. }\end{array}$ & $\begin{array}{l}\text { Should my composite } \\
\text { restorations last forever? Why are } \\
\text { they failing? }\end{array}$ & $\begin{array}{c}\text { Apresentar uma visão geral da literatura recente } \\
\text { em relação ao desempenho clínico } \\
\text { de restaurações compostas diretas em dentes e } \\
\text { discutir os principais fatores que afetam a } \\
\text { longevidade. }\end{array}$ & $\begin{array}{l}\text { Revisão } \\
\text { Bibliográfic } \\
\quad \text { a }\end{array}$ & 2017 \\
\hline $\begin{array}{l}\text { GRESNIGT, Marco } \\
\text { M.M. et al. }\end{array}$ & $\begin{array}{l}\text { Comparison of conventional } \\
\text { ceramic laminate veneers, partial } \\
\text { laminate veneers and direct } \\
\text { composite resin restorations in } \\
\text { fracture strength after aging }\end{array}$ & $\begin{array}{l}\text { Testar a resistência à fratura in vitro de facetas } \\
\text { laminadas e restaurações compostas após } \\
\text { envelhecimento e analisar o modo de falha. }\end{array}$ & $\begin{array}{c}\text { Pesquisa } \\
\text { Laboratorial }\end{array}$ & 2021 \\
\hline $\begin{array}{l}\text { KOCAAĞAOĞLU, H } \\
\text { et al. }\end{array}$ & $\begin{array}{l}\text { Efficacy of Polishing Kits on the } \\
\text { Surface Roughness and Color } \\
\text { Stability of Different Composite } \\
\text { Resins }\end{array}$ & $\begin{array}{l}\text { Avaliar a rugosidade da superfície e estabilidade } \\
\text { de cor de quatro compósitos diferentes aos quais } \\
\text { foi aplicado polimento diferente técnica. }\end{array}$ & $\begin{array}{c}\text { Pesquisa } \\
\text { Laboratorial }\end{array}$ & 2021 \\
\hline $\begin{array}{c}\text { D'ONOFRE, Pedro } \\
\text { Lucas et al. }\end{array}$ & $\begin{array}{l}\text { A faceta de resina composta } \\
\text { direta como uma técnica } \\
\text { restauradora minimamente } \\
\text { invasiva para harmonizar o } \\
\text { sorriso. }\end{array}$ & $\begin{array}{l}\text { Relatar um caso clínico, no qual a técnica } \\
\text { cirúrgica direta das facetas estéticas em resina } \\
\text { composta foi utilizada como alternativa para o } \\
\text { tratamento restaurador de dentes anteriores, após } \\
\text { tratamento ortodôntico insatisfatório do ponto de } \\
\text { vista estético. }\end{array}$ & $\begin{array}{l}\text { Relato de } \\
\text { Caso }\end{array}$ & 2020 \\
\hline $\begin{array}{l}\text { GOUVEIA, Camilla } \\
\text { Gonzaga et al. }\end{array}$ & $\begin{array}{c}\text { Facetas diretas de resina } \\
\text { composta em dentes anteriores: } \\
\text { relato de caso. }\end{array}$ & $\begin{array}{l}\text { Abordar a troca de restaurações comprometidas } \\
\text { esteticamente com auxílio de modelos de estudo } \\
\text { associados ao enceramento diagnóstico e guia de } \\
\text { silicone, na confecção de restaurações diretas de } \\
\text { resina composta nos dentes superiores. }\end{array}$ & $\begin{array}{l}\text { Relato de } \\
\text { Caso }\end{array}$ & 2018 \\
\hline $\begin{array}{l}\text { AQUINO, José } \\
\text { Milton et al. }\end{array}$ & $\begin{array}{l}\text { Restabelecimento funcional e } \\
\text { estético utilizando as facetas } \\
\text { na odontologia moderna }\end{array}$ & $\begin{array}{l}\text { Analisar a importância da utilização das facetas } \\
\text { de forma minimamente invasiva nos tratamentos } \\
\text { odontológicos. }\end{array}$ & $\begin{array}{c}\text { Revisão } \\
\text { Bibliográfic } \\
\text { a }\end{array}$ & 2021 \\
\hline
\end{tabular}

Fonte: Autores (2021). 
Como mostra a tabela acima, dentre os artigos selecionados e analisados, todos apresentaram conteúdos relativos a facetas direta anteriores, incluindo suas vantagens e limitações. Em relação ao tipo de pesquisa todos os artigos tratava-se de relatos de casos. Teve-se uma variação concernente ao ano de publicação, onde os artigos foram publicados entre 2016 e 2019.

Segundo o estudo de caso realizado por Baptista e colaboradores (2019) sobre associação de diferentes cirurgias periodontais como alternativa de restabelecimento de harmonia gengival, existem diversas alternativas para tratamentos estéticos dos quais o cirurgião-dentista pode lançar mão. Dentre estas, estão as técnicas indiretas coroas totais e as facetas de porcelana, as quais possuem capacidade funcional e estética excelentes. Contudo, essas técnicas são conceituadas como invasivas e de custo elevado. Em contra partida, o protocolo adesivo de facetamento direto com resina composta tem se tornado bastante conhecido atualmente, por ser uma técnica minimamente invasiva e de custo menor, quando comparada às facetas indiretas.

Contudo, em comparação com os laminados cerâmicos tradicionais, as facetas diretas em resina composta podem reduzir a remoção do tecido. Além disso, o tratamento direto pode ser realizado em um único atendimento e é relativamente barato. No entanto, as restaurações diretas de resina composta têm suas desvantagens: elas precisam ser substituídas devido ao desgaste e à perda da forma anatômica e não mantem estabilidade de cor em longo prazo. Além das mudanças na cor e no desgaste do material compósito, existe outro fator que pode causar o fracasso da restauração composta. Embora as facetas diretas com resina seja um tratamento mais barato, eles não apresentam estabilidade de cor e apresentam alto índice de desgaste. As facetas de cerâmica não são afetadas por esses fatores, mas são uma opção mais invasiva. Ambos os tipos de restaurações estão sujeitos a fraturas (Gresnigt, Sugii, Johanns, \& Made, 2021).

Gouveia et al. (2018), no estudo reiteram que ao restaurar os dentes anteriores, é necessário a escolha dos materiais corretos, como: tipos de resinas, sistemas adesivos e ótimos fotopolimerizadores, pois os mesmos são essenciais para a qualidade da técnica. Para casos que não necessite de pouco ou até nenhum preparo, as facetas diretas resinas compostas são uma excelente escolha para correção ou remodelação estética dos dentes anteriores. Em um estudo, é possível observar um índice de $84 \%$ de vida útil dos parâmetros qualificados da resina composta após cinco anos tratamento, mas por ser de fácil reparo, a taxa de sobrevida funcional pode ser considerada em $100 \%$.

Aquino et al. (2021) no seu estudo abordam que maioria dos casos, a contraindicação é estabelecida em elementos dentais que não possuem suporte saudável em especial nas estruturas que envolve o esmalte, pacientes com bruxismo não tratado, e também aqueles que tem o hábito de ranger os dentes, outras situações que contraindicam são: periodontites graves, dentes muito vestibularizados. Outros casos que possuem menos de 50\% de esmalte, dentes com múltiplas restaurações, pacientes que tem oclusão topo-a-topo, dentes com grau elevado de giroversão, mordida cruzadas, apinhamentos entre outros.

Complementando o que foi acima mencionado, Alvarenga et al. (2018) em seu trabalho aponta que, casos que necessitam de estética e/ou resistência, anteriormente só podiam ser solucionados com protocolos protéticos invasivos, atualmente são capasses de serem resolvidos com técnicas minimamente invasivas, através do uso de resinas compostas nanoparticuladas. As resinas compostas disponíveis comercialmente possibilitam restaurar os dentes mimetizando sua fluorescência e opalescência.

A partir do exposto, se pode compreender que com a evolução na qualidade dos materiais restauradores, que possibilitam agora características mais fiéis esteticamente, além de capacidade de resistir as forças mastigatórias, disponíveis no mercado, tem incentivado a confecção de facetas diretas com resina composta.

Para Kocaagaoglu et al. (2017), a mudança de cor da resina composta pode causar insatisfação de pacientes e clínicos, e o sucesso da restauração estética depende da estabilidade da cor dos materiais utilizados. O grau de mudança de cor pode ser afetado pela rugosidade da superfície, e quando a resina composta adere à matriz do filme de poliéster, a superfície mais lisa não pode ser obtida sem acabamento ou polimento. A superfície possui uma camada rica em resina e pobres propriedades 
mecânicas, que devem ser removidas. Portanto, a descoloração da resina composta pode ser eliminada. A vida útil das restaurações de resina composta é muito afetada pela qualidade dos procedimentos de acabamento e polimento. A superfície lisa reduz a retenção de placa dentária, irritação gengival, cárie dentária recorrente e descoloração da restauração. Por questões de saúde bucal, também é importante determinar o melhor tratamento de superfície / técnica de polimento para obter os melhores resultados. Por esses motivos, os protocolos de acabamento e polimento das resinas compostas são a base da prática odontológica.

D’Onofre et al. (2020) destacam que é importante dedicar tempo na fase de diagnóstico e planejamento, pois melhoram a previsibilidade e qualidade do tratamento. Além de, reduzir erros clínicos e a frustração provocados pela falha na comunicação entre profissional-paciente. Para obter resultados excelentes na reabilitação estética e funcional, todo CirurgiãoDentista deve se aprimorar no planejamento e fornecer subsídios previsíveis para os procedimentos clínicos.

Em seu estudo de caso, Machado et al. (2016) destaca que, na técnica de facetas diretas em dentes anteriores, é preciso ter atenção no momento de selecionar a resina que será utilizada, pois é fundamental que o material escolhido tenha propriedades ópticas o mais próximo possível da estrutura dentária. Para a confecção de restaurações que não sejam somente funcionais, como também estéticas, é importante ter conhecimento sobre os parâmetros de translucidez, fluorescência, cor e opalescência das resinas, facilitando o manejo dos diversos incrementos resinosos.

De acordo com Araújo et al. (2019), os compósitos resinosos atuais, possuem grande diversidade de cores e efeitos, permitindo inúmeras combinações de opacidade e translucidez, promovendo um resultado satisfatório. Os autores enfatizam ainda que, outras vantagens da técnica direta em comparação a indiretas, é que esta tem-se menor desgaste dental, tempo clínico reduzido, possibilidade de reparo, mínima agressão periodontal, evitam etapa laboratorial, não precisam de provisórios, possui rápida confecção, além de terem um custo menor. Já acerca das desvantagens em relação à técnica indireta, são difíceis de mascarar o substrato escurecido, possuem estabilidade de cor inferior e menor resistência ao desgaste, mas, ainda assim, pode se conseguir resultados satisfatórios, sempre que é bem indicada e executada de forma correta.

Nessa mesma perspectiva, Araújo et al. (2019), em seu estudo sobre reabilitação estética anterior com resina composta reforça que a indicação correta de facetas diretas de resina composta traz resultados satisfatórios, preservando à estrutura dentária e sendo uma técnica simples. Os fatores a serem considerados com a finalidade de confirmar a boa indicação são: grau de descoloração, análise oclusal, higiene do paciente, qualidade e quantidade do remanescente dental e as distâncias biológicas. Deve-se ainda, atentar a algumas condições que limitam a indicação, que são: a ausência de esmalte na cervical do dente, apinhamento severo, oclusão topo a topo e hábitos parafuncionais.

As autoras supracitadas reiteram também que, a obtenção da forma, contorno e texturas similares a estrutura dentária é desafiadora em pacientes jovens, em razão de que os dentes dessa população em específico apresentam muitos detalhes anatômicos. Nestes pacientes, a dentina é ordenada em formato de lóbulos bem evidentes e desenvolvidos, o esmalte é mais branco, com baixa translucidez e alta opalescência, já a borda incisal exibe alta translucidez e um fino halo opaco.

Diante do que foi apresentado, é notório que apesar de existirem limitações ou impossibilidades para facetamento direto, como a pouca quantidade de remanescente dental, escurecimento severo dos dentes e presença de hábitos deletérios, as facetas diretas anteriores tem sido utilizadas cada dia mais, pois, quando bem planejadas e indicadas trazem consigo vantagen s e benefícios ao paciente, tais como: estética agradável, restabelecimento fonético e funcional, além de serem mais acessíveis financeiramente quando comparadas as facetas indiretas, justificando assim, o seu uso.

Um dos grandes artifícios que pode ser utilizado e que auxilia no protocolo de confecção das facetas diretas é o planejamento reverso, feito através do enceramento diagnóstico e do ensaio restaurador intraoral. Onde, sobre o modelo de estudo acrescenta-se a cera reproduzindo condições estéticas e funcionais para o paciente, e a partir daí cria-se o mock-up. Em relação a isso, Reis et al. (2018), em seu estudo que objetivou apresentar um relato de caso clínico mostrando a importância do 
planejamento reverso na reabilitação estética, realizada por meio de facetas diretas em resina composta, enfatiza que o uso do mock-up proporciona uma simulação segura e instantânea da reabilitação final, permitindo assim, analisar o sorriso do paciente de maneira integrada aos tecidos moles, face e lábios, além da fonética e dos movimentos mandibulares.

Nesse segmento, os autores supramencionados ainda acrescentam que a técnica do mock-up é perfeitamente reversível, podendo ser diretamente executada sobre os elementos dentais com a resina bis-acrílica, a qual é composta por uma base e catalizador, que são misturados pelo sistema de ponta de auto mistura, e é quimicamente ativada. A resina bis-acrílica possui alta qualidade estética e funcional, é de fácil acabamento e polimento, possui reduzida contração de polimerização, além de excelente adesão ao elemento dental, excluindo assim, a necessidade de cimentação provisória.

Quando se trata do uso de resinas compostas nos incisivos centrais superiores, Carrijo et al. (2019), dizem que estes são mais susceptíveis a falhas e substituição. Segundo eles os dentes anteriores estão mais expostos a desafios como: traumas, desgastes na borda incisal provocadas por hábitos parafuncionais (por exemplo, bruxismo, roer unhas, apertamentos dental). A sobrecarga em pacientes com problemas oclusais provocam aumento das tensões mecânicas nas facetas diretas anteriores.

Passaram-se muitos anos até os compósitos resinosos serem considerados materiais adequados para a restauração de dentes anteriores e posteriores. A baixa resistência ao desgaste e resistência mecânica eram os defeitos da primeira geração de compósitos dentais, e o amálgama de prata da época era o material padrão ouro para restauração posterior. Com o tempo, melhorias na composição das resinas levou a melhorias significativas nas propriedades do material, agora com de monômeros com maior peso molecular e menor contração de polimerização e maior carga de partículas inorgânicas, partículas menores e morfologias diferentes. Os compostos atuais também têm maior estabilidade de cor e melhores propriedades ópticas. Neste caso, os problemas associados à escolha da resina composta, para se realizar as facetas não são mais um problema hoje (Demarco et al., 2017).

Miranda et al. (2016) em seu estudo, tratam acerca da relevância do acabamento e polimento das restaurações em resina composta, em razão de que esta etapa proporciona longevidade às restaurações, diminuindo a rugosidade, evidenciando os detalhes anatômicos e promovendo lisura e brilho superficial. O correto planejamento necessita de envolver as necessidades orais do paciente, eliminando fatores que provoquem dor e infecção, seguido de remoção de cáries e doenças periodontais, caso estas sejam necessárias, para que com o meio bucal adequado o cirurgião-dentista promova uma reabilitação estética e funcional de qualidade.

Ribeiro (2020) em seu estudo avaliou o uso da resina composta como facetas em dentes anteriores e definiu que a aplicação de restaurações diretas fornece uma excelente opção de tratamento para dentes fraturados e para fechamento de diastemas. Ainda levou em consideração as facetas diretas quando comparadas com as indiretas e chegou à conclusão que a técnica e o material apropriados para o tratamento eficaz são baseados no tempo, nas limitações físicas, psicológicas e econômicas do paciente.

Portanto, se pode inferir que um adequado planejamento, com a sequência correta do passo-a-passo clínico, com a adequação do meio bucal através da remoção de cáries, cálculos ou quaisquer outros fatores que causem desequilíbrio dental e/ou periodontal, caso estes sejam necessários, devem ser feitos, buscando garantir o sucesso da reabilitação estética e funcional por meio das facetas diretas.

Então compreende-se que, o atendimento clínico odontológico envolve diversos aspectos, tais como: situação social e econômica do paciente, especialidade e habilidade do cirurgião-dentista, fatores políticos e culturais do lugar, além da expectativa e anseios do paciente (Miranda et al., 2016).

Dessa forma, a faceta direta em resina composta é uma opção de reabilitação oral, não podendo ser meramente considerada como segunda alternativa, mas, uma possibilidade para pacientes sem condições financeiras de custear restaurações cerâmicas. Uma vez que, as resinas compostas são um bom material para a resolução de várias alterações estéticas 
e funcionais quando bem planejadas e executadas. Se considerarmos a atual realidade econômica do país, poderia até mesmo dizermos que ela, na maior parte dos casos, consistirá na primeira e única opção (Reis et al., 2018).

\section{Conclusão}

As facetas diretas em resina composta são eficientes para reabilitar elementos dentários com alterações de cor, forma e funcionalidade, permitindo estética agradável. São consideradas um procedimento conservador, de menor custo e mais rápido quando comparadas às facetas indiretas em cerâmica. Sendo necessário, no entanto, um correto planejamento, uso de materiais e instrumentais de excelente qualidade e execução criteriosa por parte do Cirurgião-Dentista.

Assim sendo sugere-se que novos estudos sejam realizados de uma maneira mais especifica com a finalidade de avaliar quantitativamente a funcionalidade e durabilidade das facetas diretas quando comparadas com as facetas indiretas, demonstrando através de dados quais são os protocolos mais seguros que garantem a funcionalidade das mesmas.

\section{Referências}

Alvarenga, D. B., Santana, C. L. V., Oliveira, F. R. D. T. S., Rodrigues, R. Q. F., Ribeiro, R. A., \& Sousa, J. N. L. (2018). Inter-relação periodontia/dentística na correção de sorriso gengival: Relato de caso clínico. Braz j periodontol, 28(2), 7-13.

Aquino, J. M., Neto, S., Miranda, T. R. S., Silva, L. T. G., \& Medeiros, M. L. B. B. (2021). Restabelecimento funcional e estético utilizando as facetas na odontologia moderna. Revista Eletrônica Acervo Saúde, 13(1), e5873-e5873.

Araújo, I. C., Melo, L. S. A. D., Teixeira, H. M., Silva, C. H. V. D., \& Menezes Filho, P. F. (2019). Restaurações estéticas em substratos com alteração cromática relato de caso. Full dent. sci, 10(40), 95-104.

Araújo, I. D. T., Abrantes, P. S., Borges, B. C. D., \& de Assunção, I. V. (2019). Reabilitação estética anterior com resina composta: Relato de caso. Revista Ciência Plural, 5(1), 89-101.

Baptista, I. A., Barbosa, I. D. P., Silva, G. M. C. D., Garcia, G. H. F., Cardia, G. S., \& Tolentino, L. D. S. (2020). Associação de diferentes cirurgias periodontais como alternativa de restabelecimento de harmonia gengival. Periodontia, 30(3), 59-68.

Biezus, G. W. (2016). Avaliação clínica de restaurações diretas com resina composta em dentes anteriores. Trabalho de Conclusão de Curso. Universidade Federal de Santa Catarina.

Carrijo, D. J., Ferreira, J. L. F., \& Santiago, F. L. (2019). Restaurações Estéticas Anteriores Diretas e Indiretas: Revisão de Literatura. REVISTA UNINGÁ, 56(S5), 1-11.

D’Onofre, P. L., Câmara, J. V. F., Paiva, R. V. de, Pereira, G. D. D. S., \& Capillé, C. L. (2020). Faceta direta em resina composta como técnica restauradora minimamente invasiva para harmonização do sorriso. Research, Society and Development, 9(8), e123985437.

Demarco, F. F., Collares, K., Correa, M. B., Cenci, M. S., Moraes, R. R., \& Opdam, N. J. (2017). Should my composite restorations last forever? Why are they failing?.Brazilian oral research, 31(suppl 1), e56.

Dos Reis Goyatá, F., Costa, H. V., Marques, L. H. G., Barreiros, I. D., Lanza, C. R. M., Júnior, J. B. N., \& Moreno, A. (2017). Remodelação estética do sorriso com resina composta e clareamento dental em paciente jovem: relato de caso. Archives of Health Investigation, 6(9), 408-413.

Gouveia, C. G., Júnior, R. M., da Silva Peralta, F., Scherma, A. P., \& de Resende, L. F. M. (2018). Facetas diretas de resina composta em dentes anteriores: relato de caso. Clínica e Pesquisa em Odontologia-UNITAU, 9(1), 44-50.

Gresnigt, M., Sugii, M. M., Johanns, K., \& van der Made, S. (2021). Comparison of conventional ceramic laminate veneers, partial laminate veneers and direct composite resin restorations in fracture strength after aging. Journal of the mechanical behavior of biomedical materials, $114,104172$.

Guerra, M. L. R. S., Venâncio, G. N., \& Augusto, C. R. (2017). Fechamento de diastemas anteriores com resina composta direta: relato de caso. Revista da faculdade de odontologia de lins, 27(1), 63-68.

Kocaagaoglu, H., Aslan, T., Gürbulak, A., Albayrak, H., Taşdemir, Z., \& Gumus, H. (2017). Efficacy of polishing kits on the surface roughness and color stability of different composite resins. Nigerian journal of clinical practice, 20(5), 557-565.

Machado, A. C., Reis, B. R., Soares, P. V., Reinke, A. C. M. A., Moura, G. F., \& Zeola, L. F. (2016). Reabilitação estética e funcional com facetas diretas após histórico de traumatismo dento-alveolar. Revista Odontológica do Brasil Central, 25(74), 154-161.

Miranda, R. R., Rizza, G. C. R., Bettero, F. C. B. S., Junior, P. C. S., \& Novais, V. R. (2016). Tratamento odontológico integrado com ênfase em estética: relato de caso. Revista Odontológica do Brasil Central, 25(74), 162-169.

Patias, R. (2015). Sobrevivência de restaurações e facetas de resina composta direta e cerâmicas em dentes anteriores: estudo retrospectivo sobre fatores relacionados à substituição e ao reparo. Dissertação de Mestrado. Universidade Federal de Pelotas.

Polit, D. F., \& Beck, C. T. (2011). Fundamentos de pesquisa em enfermagem: avaliação de evidências para a prática da enfermagem. Artmed Editora. 
Research, Society and Development, v. 10, n. 6, e48910615729, 2021

(CC BY 4.0) | ISSN 2525-3409 | DOI: http://dx.doi.org/10.33448/rsd-v10i6.15729

Pottmaier, L. F., de Azevedo Linhares, L., Sinhori, B. S., \& Bernardon, J. K. (2017). Recontorno Dental e Fechamento de Diastemas com Resina Composta: Relato de Caso. Clínica - International Journal of Brazilian Dentistry, 13(3), 288-295.

Reis, G. R., Oliveira, L. P. M., Vilela, A. L. R., \& de Sousa Menezes, M. (2018). Mock-up: Previsibilidade e facilitador das restaurações estéticas em resina composta. Revista Odontológica do Brasil Central, 27(81), 105-111.

Ribeiro, R. F. (2020). Fechamento de diastemas em dentes anteriores com laminados e resina composta. Trabalho de Conclusão de Curso. Centro Universitário São Lucas.

Santos, T. D. O. G., Donato, T. R., Mathias, C., \& Mathias, P. (2018). Avaliação radiográfica da presença de bolhas em restaurações classe II in vitro utilizando diferentes técnicas restauradoras. Revista de Ciências Médicas e Biológicas, 17(3), 381-385. 